\title{
MARY WOLLSTONECRAFT: REFERENTE FEMINISTA
}

\author{
María del Carmen SÁENZ BERCEO \\ PROFESORA TITUlAR DE HiSTORIA DEL DERECHO Y DE LAS INSTITUCIONES \\ UNIVERSIDAD DE LA RIOJA
}

SUMARIO: I. Introducción. II. Rasgos biográficos. III. Reivindicaciones. IV. Ideas, concepciones, prejuicios, mitos, que sojuzgan y esclavizan a las mujeres. IV.I. Bellas e inocentes. IV.2. Inferioridad femenina. IV.3. Esposa y madre. IV.4. Errónea o nula educación. V. Educación. VI. Conclusión.

RESUMEN: El haber nacido hombre o mujer aún es importante en relación con la posición que cada uno ocupa en el hogar, en la política, en definitiva, en la sociedad. El pertenecer a uno u otro sexo todavía condiciona las oportunidades, los trabajos, los salarios, etc. Por lo tanto, la obra de Wollstonecraft tiene hoy en día plena virtualidad y, en cierto modo, sorprende la clarividencia de esta mujer en el siglo XVIII. Se rebela contra la negación de las capacidades intelectuales de la mujer, contra la ignorancia que se le impone, y contra la imposibilidad de elección de su propio destino. Ella está convencida de que sólo si la mujer tiene una educación podrá ser independiente y le será reconocida su dignidad como mujer y como persona, y eso reclama: dignidad, educación, respetabilidad, y sobre todo, igualdad.

PALABRAS ClAVE: Igualdad, dignidad, libertad, autonomía, educación.

ABSTRACT: Having been born a man or a woman is still important today in regard to the position you have at home, in politics, and ultimately, in society. Your membership to one or another sex still determines your opportunities, your job and your salary; therefore, Wollstonecraft's work has full relevance nowadays, and in some ways, her clairvoyance is surprising coming as it does from as early as the $18^{\text {th }}$ century. She rebels against the negation of woman's intellectual capacities, against the ignorance that is imposed on her and against the impossibility of choosing her own destiny. She is convinced that only with education woman will be independent and her dignity as person will be recognised, and she demands that: dignity, education, respectability, and above all, equality.

KEYWORDS: Equality, dignity, freedom, autonomy and education.

\section{Introducción}

Mi primer contacto con Mary Wollstonecraft no fue directamente con su persona o su obra sino que la referencia vino por ser la madre de Mary Shelley, la autora de Frankenstein. Lo que no sabía en ese momento es que esa mujer había tenido una de las mentes más privilegiadas de la Europa del siglo XVIII y había sido pionera en la denuncia 
de la condición y el trato que se daba a las mujeres, en la reivindicación de los derechos de la mujer y de la igualdad entre los sexos. La distinción y desigualdad, la existente entre los hombres y las mujeres, «era tan arbitraria como las referidas al rango, la clase o los privilegios; todas aquellas que el racionalismo ilustrado había criticado e identificado» ${ }^{\mathrm{I}}$.

La revolución francesa va a suponer una gran ruptura y nuevos planteamientos en todos los ámbitos del pensamiento y de la acción. Dentro del complejo mundo de la ilustración y del primer liberalismo, se abría una puerta, tímidamente pero ya sin posible marcha atrás, respecto a la igualdad entre los sexos y su correlato social y político en cuanto a derechos y deberes. Wollstonecraft apoyaba la revolución, aunque no las matanzas que se produjeron, porque creía que los derechos humanos serían por fin reconocidos y que comenzaría a implantarse la justicia.

En I792 se publica A Vindication on the Rights of Woman, donde trata de poner en evidencia lo equivocado y pernicioso que resultan algunos de los más comunes tópicos, de las conductas y costumbres que condicionan la situación de la mujer en el país, señalando a su vez las actuaciones que permitirían superarlos y encaminar a la sociedad hacia la integración, como iguales, de quienes suponen la mitad de la humanidad. Este libro es considerado en Europa como pionero, y se le concede categoría de texto fundador en la reivindicación de los derechos de la mujer y en la lucha por la igualdad'. Es decir, que si algún trabajo ha sido significativo para poner en evidencia el injusto trato y la marginación que las concepciones tradicionales imponían a las mujeres, ha sido éste. Y en él voy a centrarme para analizar las reivindicaciones sobre los derechos de la mujer que ahí explicitó Mary Wollstonecraft. En él la autora cuestionaba las relaciones de poder: sociales, sexuales, religiosas, etc., de su época tanto como para que hoy, sus sorprendentes, y en cierto modo heterodoxas vida y obra, comiencen a resultar académica, social y editorialmente respetables.

Según sus palabras, lo que trata de hacer en ella, y que presenta en la introducción, «al desechar esas preciosas frases femeninas que los hombres usan con condescendencia para suavizar nuestra dependencia servil, y al desdeñar esa mente elegante y débil, esa sensibilidad exquisita y los modales suaves y dóciles que supuestamente constituyen las características sexuales del recipiente más frágil, deseo mostrar que la elegancia es inferior a la virtud, que el primer objetivo de una ambición laudable es obtener el carácter de un ser humano, sin tener en cuenta la distinción de sexo».

Por otro lado, aunque el haber nacido hombre o mujer ya no tiene extrema relevancia en el ámbito de lo que la ley establece para ambos, aún es importante en relacion con la posición que cada uno ocupa en el hogar, en la política, en definitiva, en la sociedad. El pertenecer a uno $u$ otro sexo todavía determina las oportunidades, los trabajos, los salarios. Las mujeres siguen discriminadas y son maltratadas y prostituidas, por lo tanto, la obra de Wollstonecraft tiene hoy plena virtualidad y, en cierto modo, sorprende la agudeza y

${ }^{\mathrm{I}}$ WOLLSTONECRAFT, M., A Vindication on the Rights of Woman, edición e introducción de Isabel Burdiel, Vindicación de los derechos de la mujer, Madrid, Cátedra, I994, pág. 58. A esta traducción y edición corresponden las referencias concretas que se hacen al libro de Wollstonecraft en este trabajo.

${ }^{2}$ LORENZO, M. J., «La vindicación de los derechos de la mujer antes de Mary Wollstonecraft», en Philologia Hispalensis, vol XVII/2, pág. Io6. 
coraje de esta mujer que expuso sus ideas hace ya casi tres siglos y que trató de vivir de acuerdo a ellas aún a riesgo de ser considerada cuanto menos excéntrica.

\section{Rasgos biográficos ${ }^{3}$}

Antes de comenzar con su obra deshilachemos el tapiz de su trayectoria vital, conozcamos algo de su vida, porque ello nos permitirá situar al personaje en su momento histórico y posiblemente comprender mejor su trabajo, ya que su vida marcó claramente la vocación de sus textos. Nos será más fácil así reconocer el mérito de una escritura desafiante con el entonces negado espacio para las mujeres.

Mary Wollstonecraft nació el 27 de abril de 1759 en Spitalfields, cerca de Londres, y murió de fiebres puerperales, o sobreparto, el Io de septiembre de I797, diez días despúes de dar a luz a su segunda hija, la futura Mary Shelley ${ }^{4}$. Fue la segunda de seis hermanos y la mayor de las chicas. Su infancia estuvo marcada por el descenso económico de la familia debido a los fallidos intentos de su padre por convertirse en un hacendado gracias a la agricultura. Para I780, cuando su madre muere, la familia había vivido en varios lugares de Inglaterra y Gales. Parece que Mary envidiaba a su hermano mayor, que era el favorito de su madre y que heredó casi toda la fortuna familiar gracias al testamento del abuelo5. Su padre era un individuo violento que maltrataba a su madre, lo que indudablemente dejará secuela en el sentir de la joven Mary.

El leit motiv de su vida, el presupuesto ineludible del que queda testimonio en varios de sus escritos, es que la mujer consiga independencia económica. Para alcanzarla, como todas las mujeres de su tiempo, educadas y pobres, Mary no tenía más que dos caminos: el matrimonio o el trabajo. Como no quería el matrimonio ocupó las profesiones que la reglas de decencia de la época permitían a las mujeres, en la idea de que esa independencia garantizaría la autonomía personal y el respeto propio y de la sociedad, y dado que prácticamente las únicas opciones eran la de dama de compañía, maestra o institutriz, desempeñó las tres. Fue dama de compañía en Bath, fundadora de una pequeña escuela en Newington Green, e institutriz en Irlanda con la familia Kingsborough, trabajo del que fue despedida. Presumiblemente ello se debió a su complicada relación con la señora Kingsborough en quien Mary veía el prototipo de mujer rica, con todos los defectos que criticará en su obra: coquetería, exagerada debilidad, corrupto y manipulador poder y a la vez dependencia mogigata de los hombres para conseguir su identidad como persona.

Afortunadamente, su amigo y editor, Joseph Johnson, la libró de caer en la indigencia o asumir un rol no deseado al contratarle como traductora, para lo que estaba

\footnotetext{
${ }^{3}$ Para elaborar su biografía se ha consultado preferentemente el trabajo de TODD, J., Mary Wollstonecraft: a speculative and dissenting spirit, que puede encontrarse en Internet:

http://www.bbc.co.uk/history/british/empire_seapower/wollstonecraft_or.shtml.

${ }^{4} \mathrm{Si}$ el destino de la mujer entonces, y todavía mucho después, era ser esposa y madre, ella cumplió esa misión y ese destino y murió de la más femenina de las formas: debido a su maternidad. Según se dijo, fue «el castigo merecido que la providencia reservaba a aquéllas que querían desembarazarse de las condiciones naturales de su sexo». POLWHELE, R., The unsex'd females, London, I798.

${ }^{5}$ Quizá ello influyó para que ella defendiera la igualdad y condenase la tradición que, bajo la supuesta naturalidad, perpetuaba la subordinación de una gran mayoría de ciudadanos mediante la jerarquía de la propiedad y los derechos adquiridos por la herencia. En I790 una ley abolía el derecho de primogenitura masculino en Francia, mientras que en España estuvo vigente hasta avanzado el siglo XIX.
} 
bien preparada ya que dominaba el inglés, el francés y el alemán, además de encargarle que escribiera y participara como editora en la revista que publicaba en Londres, Analytical Review, lo que le permitió no sólo ganarse la vida sino codearse con un vibrante círculo de intelectuales, entre los cuales pudo desarrollar sus avanzadas y críticas concepciones. Mary llegó a Londres, procedente de Irlanda, en agosto de I787, y estaba tan contenta por los nuevos derroteros que tomaba su vida que escribió a su hermana Everin «que estaba a punto de convertirse en la primera de una nueva especie».

Poco a poco se dio cuenta de que quizá lo que más le molestaba de la situación social era que los indebidamente privilegiados y educados hombres, sistemáticamente denegaban educación y autonomía a las mujeres. Durante esta época escribió sus dos, sino principales, quizá más polémicas obras. A Vindication on the Rights of Men y A Vindication on the Rights of Woman. En la primera, publicada de forma anónima en I790, cuestiona las ideas recogidas en el libro de Edmund Burke, Reflections on the Revolution in France. En la segunda, publicada en I792, y de la que nos ocupamos, son las ideas que Rousseau recoge en L'Emile sobre la educación de la mujer, las que presumiblemente suscitaron su obra. Comprendió que las mujeres, en el Contrato Social roussoniano, no nacieron libres ni encontrarían libertad dado que el argumento biologicista escribía su destino. La diferente consideración y formación de la mujer iba completamente encaminada a mantener la sumisión en la esfera patriarcal, tanto en el en el ámbito público como en el privado, en el matrimonio y en el hogar. Wollstonecraft reivindica para la nueva Sofía un rol de mujer caracterizado por la racionalidad y basado en su propia capacidad intelectual, que le llevará a la autonomía y no al sentimentalismo.

Vivió en Paris y en otros lugares de Francia. En la capital del Sena nació su primera hija, Fanny, fruto de su relación con el americano Gilbert Imlay, que no aceptó casarse con ella y la abandonó. Por encargo de éste, que era comerciante, había viajado a Escandinavia y ese viaje y esa experiencia quedaron plasmados en Letters from Sweden, que fueron publicadas en I796. Llegó a encontrar su existencia tan miserable que intentó suicidarse antes y después del mencionado viaje. El último capítulo de su vida como mujer viene marcado por dos acontecimientos relevantes: su matrimonio con el filósofo William Godwin, padre de su hija Mary, y su muerte, a consecuencia de la martenidad, con tan sólo 38 años.

\section{Reivindicaciones}

Un aspecto destacable en la obra de Wollstonecraft es su valentía, ya que se enfrentó al mundo que la rodeaba tratando de cambiarlo, pero además lo hizo desde dentro, debiendo modificar asimismo las concepciones y valores en los que había sido educada. Claro que ya se ocuparon sus contemporáneos de que los mismos fracasaran cayendo en el olvido o bien demonizándolos mediante el ridículo o el escándalo ${ }^{6}$.

\footnotetext{
${ }^{6}$ Se le dedicaron apelativos tan impactantes como «hiena con enaguas» y hemos visto hasta donde llegaban sus contemporáneos al considerar que merecía la muerte como castigo por apartarse del estereotipo femenino. Gran parte de la culpa por ello corresponde a su marido, Godwin, quien llevado de su amor por ella publicó Memoirs of Mary Wollstonecraft, en I798. En esa obra el filósofo deja al descubierto las ideas y los sentimientos más íntimos de su esposa lo que causó un tremendo impacto en la sociedad hasta el punto de que muchos que congraciaban con sus planteamientos renegaban de ello en público o simplemente mostraban su repulsa más absoluta. El libro causó un gran escándalo y desprestigió completamente a Mary Wollstonecraft. A pesar del éxito que $A$ Vindication on the Rights of Woman tuvo al publicarse, mencionar su nombre bastaba para ruborizar
} 
En A Vindication on the Rights of Woman, detalla sus concepciones y sus propuestas para colocar a la mujer en igualdad con el hombre, a la vez que se hacen presentes sus vacilaciones entre necesidad y dependencia por un lado y su incontestable anhelo de libertad y autonomía por otro. Sus ideas fueron importantes en su momento e incluso alcanzarán más relevancia a lo largo de los años. Iremos analizándolas y lo haremos en muchas ocasiones utilizando sus propias palabras, porque no es fácil superarlas ni en claridad ni en contundencia.

Su principal reivindicación, que condiciona todas las demás, es el derecho a la dignidad, que ella exige para todo el género humano, y por supuesto para la mujer, por ello espera: «que mi propio sexo me excuse si trato a las mujeres como criaturas racionales en vez de hacer gala de sus gracias fascinantes y considerarlas como si se encontraran en un estado de infancia perpetua, incapaces de valerse por sí solas. Deseo de veras señalar en qué consiste la verdadera dignidad y la felicidad humana». En aras a ese objetivo, la mujer ha de ser independiente. Por ello señala:

«Es tiempo de efectuar una revolución en los modales de las mujeres, tiempo de devolverles su dignidad perdida y hacerlas trabajar, como parte de la especie humana, para reformar el mundo, mediante su propio cambio», y continúa, «es vano esperar virtud de las mujeres hasta que no sean independientes de los hombres en cierto grado; más aun, es vano esperar esa fortaleza del afecto natural que las haría buenas esposas y madres. Mientras dependan absolutamente de sus maridos, serán arteras, ruines y egoístas; y los hombres a quienes les puede satisfacer un afecto servil semejante al del perro de aguas no tienen mucha delicadeza, porque el amor no ha de comprarse» ${ }^{7}$.

Defiende a ultranza que la mujer es una criatura con capacidad suficiente para hacerse cargo de su propio destino, que no tiene que estar subordinada a nadie, y así escribirá a Talleyrand-Périgod, antiguo obispo de Autun: «Desde hace tiempo he considerado la independencia como la gran bendición de la vida, la base de toda virtud». Reitera, dirigiéndose al obispo en su calidad de legislador,

«Que si los hombres luchan por su libertad y se les permite juzgar su propia felicidad, ¿no resulta inconsistente e injusto que subyuguen a las mujeres, aunque crean firmemente que están actuando del modo mejor calculado para proporcionarles felicidad? ¿Quién hizo al hombre el juez exclusivo, si la mujer comparte con él el don de la razón?. E impulsada por ese convencimiento exclama, Benigno Creador de toda la raza humana, ¿has creado a un ser como la mujer, que puede descubrir tu sabiduría en tus obras y sentir que solo tú eres exaltado sobre ella por la naturaleza, sin un propósito mejor? ¿Puede creer que sólo se la creó para someterse al hombre, su igual, un ser que, como ella, fue enviado al mundo para adquirir virtud? ¿Puede consentir que se la ocupe sólo en complacerlo, simplemente para adornar la tierra, cuando su alma es capaz de alzarse a ti? ¿Y puede permanecer en dependencia absoluta de la razón del hombre, cuando debe subir con él los arduos escalones del conocimiento?, y añade, debe cultivarse el entendimiento para salvar a la pobre criatura del estado frágil y dependiente que se sigue de la ignorancia inofensiva. Porque el uso adecuado de la razón es lo único que nos hace independientes de todo, excepto de la misma razón despejada, a cuyo servicio esta la libertad perfecta» ${ }^{8}$.

a quienes en algún momento habían reconocido apreciar su obra, o simplemente haberla leído. Durante todo el siglo XIX, la imagen que quedó de ella no fue precisamente la de la rígida amazona de la moral, sino la de una mujer de pasiones ingobernables; una sensualista sin refinamiento, cuyo inusual talento fue a la par de su inusual falta de delicadeza.

\footnotetext{
${ }^{7}$ WOLLSTONECRAFT, M., A Vindication on the Rights of Woman, «cit.», pág. I66.

${ }^{8}$ Ibidem, pág. 200.
} 
No admite ningún tipo de subordinación de una persona a otra, por ello se revelará contra la esclavitud. También contra la sumisión que supone el ranking social y económico en que se esté encuadrado. Casi considera la riqueza la madre de todos los vicios, al menos desde el lado femenino. En su opinión nadie, por muy pobre que sea, debe estar sometido a otro, y por supuesto, tampoco a la aristocracia, por ello rechaza la propuesta, de que debía educarse a las mujeres ricas para que ello sirviera de guía a las mujeres de clases inferiores, como sugería Catharine Macaulay y quienes más tarde fueron considerados, sino feministas, al menos, defensores de las mujeres. De la misma forma se opondrá rotundamente a la teoría de Sarah Trimmer, quien preocupada por la educación infantil sostenía que era necesario educar a las niñas pobres con el fin de que sirvieran a las mujeres de la clase alta ${ }^{\mathrm{I}}$.

La libertad es la madre de la virtud y si por su misma constitución las mujeres son esclavas y no se les permite respirar el aire vigoroso de la libertad, ¿deben languidecer por siempre y ser consideradas como exóticas y hermosas imperfecciones de la naturaleza? Y lanza a sus contemporáneos una serie de cuestiones en relación con la mujer, y así les interroga: «¿Por qué se le limita, por qué no se le educa, por qué se le margina, por qué no accede al trabajo como los hombres?» Y si su destino es ser esposa y madre «¿Posee suficiente carácter para ocuparse de una familia o educar a sus hijos la mujer que desde muy pronto ha asimilado nociones de obediencia pasiva?».

Su respuesta es categórica y a la vez triste y así señala: «Tras investigar la historia de la mujer, hasta ahora no puedo evitar coincidir con los críticos más severos y considerar a nuestro sexo el mas débil, así como la mitad mas oprimida de la especie». Lucha por la respetabilidad y tratando de explicar su actitud insiste:

«Quiero persuadir a las mujeres para que traten de conseguir fortaleza, tanto de mente como de cuerpo, y convencerlas de que las frases suaves, el corazón impresionable, la delicadeza de sentimientos y el gusto refinado son casi sinónimos de epítetos de la debilidad, y que aquellos seres que son sólo objetos de piedad y de esa clase de amor que se ha calificado como su gemela pronto se convertirán en objetos de desprecio»" ${ }^{\text {II }}$.

Y para concienciar a sus congéneres pone en evidencia la maldad del sistema, dado que: «La debilidad puede excitar la ternura y satisfacer el orgullo arrogante del hombre; pero las caricias condescendientes de un protector no gratificaran a una mente noble que anhela y merece ser respetada». Por ello, considera imprescindible hacerse respetable y ejercitar el entendimiento, ya que no hay ningún otro fundamento para obtener un carácter independiente; «quiero decir explícitamente que las mujeres sólo deben doblegarse a la autoridad de la razón, en lugar de ser las modestas esclavas de la opinión».

\footnotetext{
${ }^{9}$ Letters on Education, (1790).

Io Reflections on the Education, (I792).

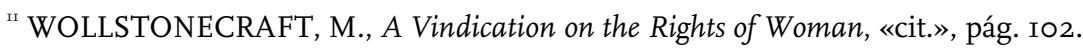




\section{Ideas, concepciones, prejuicios, mitos, que sojuzgan y esclavizan a las mujeres}

\section{IV.r. Bellas e inocentes}

Ese era el canon de glamour de la época. El mantenimiento de la belleza y la obediencia ciega al hombre se decía que eran las mejores prendas de una mujer. A Wollstonecraft le parece inadmisible que en la sociedad a la que pertenece los conocimientos y comportamientos que se esperan de una mujer sean afectados, mezclados con un absurdo e irreal romanticismo que señala que su poder está en la belleza (corporal), en la seducción, en definitiva, en el vicio. John Milton se hace eco de esas concepciones cuando Eva, modelo de belleza, se dirige a Adán de esta forma.

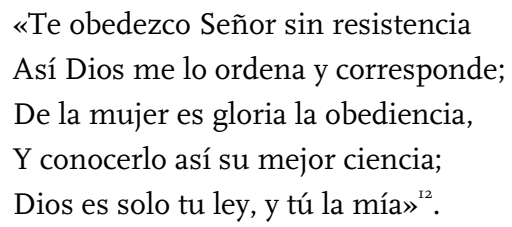

Contra todo ello arremeterá Wollstonecraft y tratando de despertar a las mujeres les arenga:

«Hermanas mías, [...] tenéis que adquirir esa sobriedad mental que sólo inspira la ejecución de las obligaciones y la búsqueda de conocimiento o permaneceréis en una situación dependiente de duda y sólo se os amará mientras seáis hermosas! [...]

Las mujeres civilizadas de nuestro siglo, con unas pocas excepciones, sólo desean fervientemente inspirar amor, cuando debieran abrigar una ambición más noble y exigir respeto por su capacidad y sus virtudes [...] Deben ser conscientes de que el poder ilegítimo que obtienen al degradarse es una maldición y de que deben volver a la naturaleza y la igualdad. La debilidad, la inocencia que tanto se predica del bello sexo, no es más que una forma cortés e interesada de llamar a la ignorancia. Se nos aconseja desde niñas convertirnos sólo en animales gentiles y domésticos, ¡de qué modo tan grosero nos insulta quien así nos aconseja! [...] Pero sea amada o descuidada, su primer deseo debe consistir en hacerse respetable y no depender para toda su felicidad de un ser sujeto a sus mismas debilidades» ${ }^{13}$.

Ella lo ve claro, pero quizá tratando de ser menos combativa y admitiendo que la mujer debe ser bella e inocente para convertirla en una compañía más atractiva y condescendiente, no puede evitar que sus ideas afloren y se pregunta: «¿Por qué se sacrifica su entendimiento? ¿Por qué ha de ser además necia?».

\section{IV.2. Inferioridad femenina}

Verdad casi revelada y documentada científicamente. Podemos admitir que en todo el mundo, y desde luego en la sociedad inglesa, enormemente puritana, se consideraba que la mujer era inferior al hombre. Era uno de los grandes tópicos que se mantenían sin discusión. Wollstonecraft entra a cuestionar el asunto, y aún aceptándolo, insiste en que los hombres han aumentado esa inferioridad hasta situar a las mujeres casi por debajo de la categoría de criaturas racionales, por ello reclama:

\footnotetext{
${ }^{\mathrm{I} 2}$ MILTON, J., El paraíso perdido, HERMIDA, B. R., (Trad.), Madrid, I8I4, pág. I63.

${ }^{\mathrm{I} 3}$ WOLLSTONECRAFT, M., A Vindication on the Rights of Woman, «cit.», pág. I64.
} 
«Dejemos espacio a sus facultades para que se desarrollen y que sus virtudes se fortalezcan [...] Cultivemos sus mentes, démosles el freno saludable y sublime de los principios y permtitámosles obtener una dignidad consciente al sentirse sólo dependientes de Dios. Enseñémosles, en común con los hombres, a someterse a la necesidad, en lugar de dar un sexo a la moral para hacerlas más placenteras. Mas aún, si la experiencia demuestra que no pueden lograr el mismo grado de fortaleza mental, perseverancia y entereza, dejemos que sus virtudes sean de la misma clase, aunque luchen vanamente para obtener el mismo grado; y la superioridad del hombre estará igualmente clara, si no más» ${ }^{\mathrm{T}}$.

Los hombres poseen una fortaleza corporal superior pero si no fuera por las erróneas nociones de belleza, las mujeres adquirirían la suficiente para ser capaces de ganar su propio sustento, verdadera definición de la independencia, y para soportar aquellos inconvenientes y tareas corporales que son requisito para fortalecer la mente. En su razonamiento, contundente si se analiza despacio, «si tanto el hombre como la mujer comparten la misma capacidad racional que deriva de Dios, excepto la diferencia física, todas las distinciones entre los sexos son resultado de convenciones sociales y diferencias en la educación para hombres y mujeres».

\section{IV.3. Esposa y madre}

El matrimonio se veía como una tabla de salvación contra la miseria, y a veces, contra la pérdida de status, tal y como deja ver claramente Jane Austen cuando refiriéndose a Carlota escribe que ella «nunca había formado un gran concepto de los hombres ni del matrimonio, pero siempre quiso casarse; no había otra salida honrosa para una joven bien educada y con escasos medios de fortuna. Si no era seguro que el matrimonio hiciese feliz a una mujer, era, sin embargo, el remedio más agradable contra la pobreza» ${ }^{15}$.

Por el contrario, Wollstonecraft no era partidaria del mismo, alejándose del pensamiento y del destino casi exclusivo para la mujer en su tiempo. No compartía esa idea, y de hecho, fue tardíamente, y quizá para salvar la situación de su hija en la tradicional y conservadora Inglaterra, cuando contrajo matrimonio al saber que estaba embarazada. El destino que la naturaleza había ideado para la mujer, y para lo que había de prepararse, era para ser esposa y madre. Ella admite a regañadientes ese destino natural de la mujer pero no suscribe que sea el único. Reclama que el primer deber de toda mujer es hacia ella misma, como criatura racional, y a continuación en cuanto a importancia, como ciudadana, está el de madre, que incluye muchos otros. Y así mantiene que:

«Se ha de conducir a las mujeres a respetarse, han de abrirse para ellas los temas políticos y morales como el único medio de conseguir que atiendan apropiadamente sus obligaciones domésticas, porque una mente activa abarca todo el círculo de sus deberes y encuentra tiempo suficiente para todos. Porque, ¿ha nacido la mujer solo para procrear y morir? ¿Cómo puede una mujer falta de reflexión educar a sus hijos? ¿Cómo puede inclinarlos hacia las virtudes que desconoce, o hacia el mérito del que no tiene idea? Sólo puede consolarlos o reprenderlos; hacerlos insolentes o tímidos; los hará fanfarrones o zopencos ignorantes, pero nunca logrará hacerlos juiciosos o amables, porque en definitiva, sería tan sabio esperar maíz de la cizaña o peras del olmo, como esperar que una mujer necia e ignorante sea una buena madre» ${ }^{16}$.

\footnotetext{
${ }^{\text {I4 }}$ Ibidem, pág. I52.

${ }^{15}$ AUSTEN, Jane, Pride and prejudice, London, Penguin, I995, cap. 22.

${ }^{16}$ WOLLSTONECRAFT, A Vindication on the Rights of Woman, «cit.», pág. 232.
} 
Se rebela contra la ignorancia impuesta a la mujer, contra la negación de la posibilidad de la razón y las diferencias de raciocinio entre los sexos, y defiende que tanto el hombre como la mujer comparten la misma capacidad racional que deriva de Dios. En su sentir, quien puede pensar correctamente pronto extenderá su imperio intelectual; «y la mujer que tenga el juicio suficiente para dirigir a sus hijos no se someterá , esté acertada o equivocada, al de su marido, o a las leyes sociales que hacen de la esposa una nulidad».

Reitera que si ser esposa y madre es el destino que la naturaleza reserva a las mujeres, se las ha de educar para que puedan cumplirlo.

\begin{abstract}
«Como se ha insistido en que el destino propio de la mujer es la crianza de los hijos, esto es, el establecimiento de los cimientos para que la generación en ciernes posea una salud sólida tanto de cuerpo como de alma, la ignorancia que las incapacita resulta contraria al orden de las cosas. Y sostengo que sus mentes pueden dar mucho mas de sí o nunca se convertirán en madres juiciosas [...] por ello si los hombres rompieran con generosidad nuestras cadenas y se contentaran con la camaradería racional en lugar de la obediencia servil, hallarían en nosotras hijas más obsequiosas, hermanas más afectuosas, esposas más fieles y madres más juiciosas; en una palabra, mejores ciudadanas. Entonces los amaríamos con afecto verdadero, porque aprenderíamos a respetarnos a nosotras mismas, y la paz mental de un hombre valioso no sería perturbada por la necia vanidad de su esposa, ni los niños se irían a cobijar a un pecho extraño, al no haber encontrado nunca un hogar en el de su madre» ${ }^{17}$.
\end{abstract}

No se resigna y repite que las mujeres podrían estudiar el arte de curar y ser médicas y enfermeras, o formarse en otros ámbitos y de este modo podrían dedicarse a tareas muy diferentes si se las educara de manera más ordenada, lo que salvaría a muchas de la prostitución común y de la legal. Puede parecer fuerte o demasiado efectista esta terminología, pero ella considera que el matrimonio, única tabla de salvación para conseguir el sustento de la mujer, es en realidad, tal y como estaba planteado, con total sometimiento de la mujer al marido, su ignorancia, su indefensión, etc. una prostitución legal y así insiste: «Es cierto que se les proporciona alimento y ropa sin que se esfuercen o tengan que dar vueltas; pero a cambio entregan salud, libertad y virtud». Señala que todo ello se debe, en buena parte, al estado de indolencia en el que se educa a las mujeres, a las que siempre se enseña a buscar un hombre que las mantenga y a considerar sus personas la recompensa adecuada por los esfuerzos para mantenerlas, y exclama casi con rabia:

« Cuántas mujeres malgastan de este modo sus vidas, presas del descontento, cuando podían haber trabajado como médicas, haber regido una granja o dirigido una tienda, y mantenerse erguidas, sostenidas por su propia industria, en lugar de bajar las cabezas sobrecargadas con el rocío de la sensibilidad, que consume la belleza a la que al principio da lustre! ¡Cuánto más respetable es la mujer que se gana su pan cumpliendo un deber que la belleza más lograda!».

Continuando la comparación, recalca que a los hombres se los prepara en su juventud para distintas profesiones, sin considerar el matrimonio el gran acontecimiento de sus vidas, mientras que las mujeres, por el contrario, no tienen otro proyecto para agudizar sus facultades.

\footnotetext{
${ }^{17}$ Ibidem, pág. 325.
} 
IV.4. Errónea o nula educación

Esa es en su opinión la principal causa de la situación de la mujer como pone de manifiesto cuando declara que tiene la profunda convicción:

«De que la educación descuidada de mis semejantes es la gran fuente de la calamidad que deploro y de que a las mujeres, en particular, se las hace débiles y despreciables por una variedad de causas concurrentes, originadas en una conclusión precipitada». E insiste: «La instrucción que han recibido las mujeres hasta ahora sólo ha tendido a convertirlas en objetos insignificantes del deseo ¡meras propagadoras de necios!, si puede probarse que al pretender adiestrarlas sin cultivar sus entendimientos se las saca de la esfera de sus deberes y se las hace ridículas e inútiles cuando pasa el breve florecimiento de la belleza, doy por sentado que los hombres racionales me excusarán por intentar persuadirlas para que se vuelvan más masculinas y respetables» ${ }^{18}$.

Y más adelante, quizá con un sesgo burlón, añade: «La misma sucesión de pensamiento me ha llevado a imaginar que las pocas mujeres extraordinarias que han corrido por direcciones excéntricas, fuera de la órbita prescrita para su sexo, eran espíritus masculinos confinados por error en estructuras femeninas». Se refiere Wollstonecraft a la realidad imperante al respecto no sólo en la sociedad inglesa sino en toda Europa, de que a la mujer que pretendía aprender, tener mejor educación, trabajar y ganarse la vida sin depender de nadie, se le consideraba una anormalidad de la naturaleza, marisabidilla, entrometida, mitad masculina, y por ende, muy poco femenina, porque ya se sabe, «fue creada para ser juguete del hombre, su sonajero, y debe cascabelear en su oído cuando quiera que, desechando la razón, le apetezca divertirse. Se las hizo para ser amadas y no deben aspirar al respeto, si no quieren ser acosadas por la sociedad como masculinas». Pero ella no se arredra y a pesar de esos apelativos casi insultantes recomienda a las mujeres: «Sed siempre prudentes en demostrar vuestro buen juicio, pues se pensará que asumís una superioridad sobre el resto de los presentes. Y si por casualidad tenéis algún conocimiento, guardarlo como un profundo secreto, especialmente de los hombres, que en general miran con ojos celosos y malignos a una mujer de gran talento y entendimiento cultivado».

Exige que se eduque a la mujer en los mismos principios, en las mismas técnicas y en los mismos conocimientos que a los hombres. No se negaba a las damas el derecho a ser educadas pero se mantenía que su educación debía ser doméstica, mientras que al hombre se le había de formar para los negocios, para los grandes asuntos y para la política. Por ello, a las mujeres se les enseñaban las nociones básicas de lectura, escritura y labores del hogar, mientras que a los hombres se les educaba en las matemáticas, la economía, las ciencias, la historia, etc. Ella pide igualdad de trato y de educación para mujeres y hombres, que no haya diferencias, que se eduquen juntos y de la misma manera.

Ahora bien, esa enorme diferencia a la hora de plantearse la educación para hombres y mujeres, ya hemos señalado que no se daba sólo en Inglaterra. ¿Qué ocurría por ejemplo en España?

En nuestro país, La ley de Instrucción Pública de 9 de septiembre de 1857, la denominada como Ley Moyano, mantenía sesenta y cinco años más tarde, enseñanzas diferentes y educación físicamente separada para ambos sexos, aunque, como norma considerada avanzada, al menos recogía que se establecieran escuelas para niñas. Ahora

\footnotetext{
${ }^{18}$ Ibidem, pág. I04.
} 
bien, las breves nociones de Agricultura, Industria y Comercio, los principios de Geometría, Dibujo Lineal y de Agrimensura, así como las nociones generales de Física y de Historia Natural, que se establecían para los chicos, serían reemplazadas en el caso de las niñas por: Labores propias de su sexo, Elementos de Dibujo aplicado a las mismas labores, y por ligeras nociones de Higiene doméstica. ¿Y cuáles eran las labores propias del sexo femenino? Según se especificaba en el artículo ig8 del Real Decreto de 16 de febrero de 1825 , eran: «hacer calceta, cortar y coser las ropas comunes de uso, bordar y hacer encajes u otras que suelen enseñarse a las niñas» ${ }^{\text {19 }}$.

\section{Educación}

Wollstonecraft está convencida de que la única vía posible para superar esa situación de la mujer es la educación, que sólo si se le educa podrá ser independiente y le será reconocida su dignidad como mujer y como persona:

«Al luchar por los derechos de la mujer, mi argumento principal se basa en este principio fundamental: si no se la prepara con la educación para que se vuelva la compañera del hombre, detendrá el progreso del conocimiento y la virtud; porque la virtud debe ser común a todos o resultará ineficaz para influir en la práctica general [...]

Las mujeres consideradas no sólo criaturas morales, sino también racionales deben tratar de adquirir las virtudes (perfecciones) humanas por los mismos medios que los hombres, en lugar de ser educadas como una especie de fantásticos seres a medias».

Claro que teniendo en cuenta que sólo la educación que enseña a los jóvenes cómo comenzar a pensar merece llamarse cultivo mental.

Ella está convencida de que la única solución para superar la desigualdad, el sometimiento y menosprecio de la mujer es la educación, y así:

«Al luchar por los derechos de la mujer, mi argumento principal se basa en este principio fundamental: si no se la prepara con la educación para que se vuelva la compañera del hombre, detendrá el progreso del conocimiento y la virtud; porque la virtud debe ser común a todos o resultará ineficaz para influir en la práctica general [...]

Las mujeres consideradas no sólo criaturas morales, sino también racionales deben tratar de adquirir las virtudes (perfecciones) humanas por los mismos medios que los hombres, en lugar de ser educadas como una especie de fantásticos seres a medias».

$[\ldots]$

Si el matrimonio es el fundamento de la sociedad, todo el género humano debe educarse según el mismo modelo o la relación entre los sexos nunca merecerá el nombre de camaradería, ni las mujeres cumplirán las obligaciones propias de su sexo, hasta que se conviertan en ciudadanas ilustradas, hasta que sean libres al permitírseles ganar su propio sustento e independientes de los hombres [...] del mismo modo que un hombre es independiente de otro», e insiste, «el matrimonio nunca se conservará como algo sagrado hasta que las mujeres, al ser criadas con los hombres, estén preparadas para ser sus compañeras en lugar de sus concubinas» ${ }^{20}$.

${ }^{19}$ Real Decreto aprobando el Plan $y$ Reglamento de las Escuelas de Primeras Letras. Gaceta de Madrid, n 23 , de 22 de febrero de 1825 .

${ }^{20}$ WOLLSTONECRAFT, A Vindication on the Rights of Woman, «cit.», pág. 35 I. 


\section{Conclusión}

Igualdad y dignidad para la mujer, a través de la educación, porque ello traerá todo lo demás.

«No veremos mujeres afectuosas hasta que se establezca una mayor igualdad en la sociedad; hasta que se confundan los rangos y sean libres, no veremos esa felicidad doméstica dignificada, cuya grandeza sencilla no pueden disfrutar las mentes ignorantes o viciadas [...] La razón y la experiencia me convencen de que el único método para conducir a las mujeres a cumplir sus obligaciones particulares es librarlas de todo freno al permitirles participar de los derechos inherentes al género humano» ${ }^{21}$.

Ahora bien, en todo ese proceso las mujeres no están, ni deben estar solas, por ello sugiere: «Hagamos a las mujeres criaturas racionales y ciudadanas libres, y rápidamente se volverán buenas esposas y madres, esto es, si los hombres no descuidan los deberes de maridos y padres». No es una ilusa, sabe que nada cambiará si los hombres no se implican en el proceso y si bien es verdad que a medida que la política sana vaya difundiendo la libertad, la humanidad, incluidas las mujeres, se hará mas sabia y más virtuosa, ese es un camino a recorrer conjuntamente con los varones, pues todo ello afecta por igual a unas y a otros.

Las ideas de Mary Wollstonecraft son ya patrimonio de la humanidad; sus pensamientos, sus planteamientos, sus exigencias, pueden descubrirse y reinventarse todos los días, porque no, no es cierto que estemos en igualdad de condiciones con los hombres, creo que todavía falta mucho por conseguir.

\footnotetext{
${ }^{21}$ Ibidem, pág. 366.
} 